\title{
Gut microbial profile is altered in primary biliary cholangitis and partially restored after UDCA therapy
}

\author{
Ruqi Tang, ${ }^{1}$ Yiran Wei, ${ }^{1}$ Yanmei Li, ${ }^{1}$ Weihua Chen, ${ }^{1}$ Haoyan Chen, ${ }^{1}$ Qixia Wang, ${ }^{1}$ \\ Fan Yang, ${ }^{1}$ Qi Miao, ${ }^{1}$ Xiao Xiao, ${ }^{1}$ Haiyan Zhang, ${ }^{1}$ Min Lian, ${ }^{1}$ Xiang Jiang, ${ }^{1}$ \\ Jun Zhang, ${ }^{1}$ Qin Cao, ${ }^{2}$ Zhuping Fan, ${ }^{2}$ Maoying Wu, ${ }^{3}$ Dekai Qiu, ${ }^{1}$ Jing-Yuan Fang, \\ Aftab Ansari, ${ }^{4}$ M Eric Gershwin, ${ }^{5}$ Xiong Ma ${ }^{1}$
}

Additional material is published online only. To view please visit the journal online (http://dx.doi.org/10.1136/ gutjnl-2016-313332).

For numbered affiliations see end of article.

\section{Correspondence to} Professor Xiong Ma, Renji Hospital, School of Medicine, Shanghai Jiao Tong University, Shanghai Institute of Digestive Disease, 145 Middle Shandong Road, Shanghai 200001, China; maxiongmd@hotmail.com

RT, YW and YL joint first coauthorship

RT, MEG and XM joint last coauthorship

Received 31 October 2016 Revised 28 January 2017 Accepted 30 January 2017 Published Online First 17 February 2017
Check for updates

To cite: Tang R, Wei Y, Li Y, et al. Gut 2018:67:534-571.

\section{ABSTRACT}

Objective A close relationship between gut microbiota and some chronic liver disorders has recently been described. Herein, we systematically performed a comparative analysis of the gut microbiome in primary biliary cholangitis (PBC) and healthy controls.

Design We first conducted a cross-sectional study of 60 ursodeoxycholic acid (UDCA) treatment-naïve patients with PBC and 80 matched healthy controls. Second, an independent cohort composed of 19 treatment-naive patients and 34 controls was used to validate the results. Finally, a prospective study was performed in a subgroup of 37 patients with PBC who underwent analysis before and after 6 months of UDCA treatment. Faecal samples were collected, and microbiomes were analysed by 165 ribosomal RNA gene sequencing. Results A significant reduction of within-individual microbial diversity was noted in PBC $(p=0.03)$. A signature defined by decreased abundance of four genera and increased abundance of eight genera strongly correlated with PBC (area under curve $=0.86$, 0.84 in exploration and validation data, respectively). Notably, the abundance of six PBC-associated genera was reversed after 6 months of UDCA treatment. In particular, Faecalibacterium, enriched in controls, was further decreased in gp210-positive than gp210-negative patients $(p=0.002)$. Of interest was the finding that the increased capacity for the inferred pathway, bacterial invasion of epithelial cells in PBC, highly correlated with the abundance of bacteria belonging to

Enterobacteriaceae.

Conclusions This study presents a comprehensive landscape of gut microbiota in PBC. Dysbiosis was found in the gut microbiome in PBC and partially relieved by UDCA. Our study suggests that gut microbiota is a potential therapeutic target and diagnostic biomarker for PBC.

\section{INTRODUCTION}

Primary biliary cholangitis (PBC), formerly known as primary biliary cirrhosis, is the most common autoimmune liver disease, ${ }^{1}$ and occurs in approximately $0.1 \%$ of women over the age of $40 .^{2} \mathrm{PBC}$ is characterised by the presence of antimitochondrial antibodies (AMA) and destruction of intrahepatic small bile ducts, leading to cholangitis, fibrosis and potentially cirrhosis. ${ }^{3}$ Treatment with ursodeoxycholic acid (UDCA) is standard for PBC, and can improve liver functional tests and slow the progression of disease. ${ }^{4}$

\section{Significance of this study}

What is already known on this subject?

- Primary biliary cholangitis (PBC) is a chronic autoimmune liver disorder that is triggered by environmental factors in genetically susceptible individuals.

- Ursodeoxycholic acid (UDCA) can improve biochemical liver tests, delay disease progression and prolong survival in PBC.

- Increasing evidence suggested that gut microbiota plays a critical role in influencing predisposition to chronic liver disorders.

\section{What are the new findings?}

- The gut microbiome of UDCA treatment-naive PBC reflected reduced species richness and a distinct overall microbial diversity, compared with healthy controls.

- A microbial profile characterised by changes in 12 genera is associated with PBC.

- The microbial dysbiosis was partially ameliorated by UDCA treatment.

- Imputed metagenomic analysis revealed perturbations of functional modules in the gut microbiomes of PBC.

How might it impact on clinical practice in the foreseeable future?

- These data suggest the potential of gut microbiota as a novel diagnostic biomarker and therapeutic target in PBC.

PBC is thought to be triggered by environmental factors in genetically susceptible individuals. ${ }^{5}$ Genome-wide association and murine model studies have expanded our knowledge of $\mathrm{PBC}$, but the pathogenesis of disease remains obscure. ${ }^{6-9}$

Recent progress in understanding of the composition and function of human microbiota reveals the important role of microbiota in immune homeostasis. ${ }^{10-12}$ It is increasingly recognised that the composition of the gut microbiota plays a critical role in influencing predisposition to chronic liver disorders including non-alcoholic fatty liver disease, alcoholic liver disease, cirrhosis and primary sclerosing cholangitis (PSC). ${ }^{13-16}$ Since the liver receives 
most of its blood supply from the intestine, it is exposed to the microbes and microbial products of the gut. In pathological conditions, perturbations to the microbiota can increase intestinal permeability leading to bacterial translocation and, in turn, trigger chronic hepatic inflammation and fibrosis. ${ }^{17}{ }^{18}$ Conversely, bile acids that are important metabolites of the microbiome can modulate the composition of the gut microbiota directly or indirectly through activation of the innate immune system. ${ }^{19}{ }^{20} \mathrm{~A}$ recent study characterised the gut microbiome in PBC using a relatively small cohort, resulting in insufficient statistical power to determine within-individual or between-individual diversities. ${ }^{21}$ The results were also likely affected by the use of UDCA.

We report herein substantial differences in the composition and function of the gut microbiome between treatment-naive PBC subjects and healthy controls. Furthermore, to examine the effect of UDCA intervention on gut microbiota in PBC, we compared the microbial communities in $\mathrm{PBC}$ before and after a 6-month course of UDCA therapy. The results obtained indicate that UDCA treatment partially ameliorated the dysbiosis. This study presents a comprehensive picture of the microbiota in $\mathrm{PBC}$ and offers a novel insight into robust biomarkers that can assist in future studies of dysbiosis.

\section{METHODS}

Study cohort

There were three study groups. We first conducted a crosssectional study of 60 UDCA treatment-naïve PBC and 80 matched healthy controls. Second, an independent cohort composed of 19 treatment-naïve patients and 34 controls was used to validate the results. Finally, a prospective study was performed in a subgroup of 37 patients with PBC who underwent analysis before and after 6 months of UDCA. All patients with PBC were recruited from the outpatient clinic of Renji Hospital affiliated to the School of Medicine, Shanghai Jiao Tong University and diagnosed according to the criteria of the American Association for the Study of Liver Diseases. ${ }^{22}$ The diagnosis of PBC was based on the following three criteria: (1) biochemical evidence of cholestasis (elevation of alkaline phosphatase (AKP) in serum); (2) a positive test for AMA and (3) a liver biopsy with histological evidence of non-suppurative destructive cholangitis and destruction of interlobular bile ducts. Patients who fulfilled at least two of the three criteria were included in the study. No patients were diagnosed as PBC-autoimmune hepatitis (AIH) overlap according to the PARIS criteria. ${ }^{23}$ All patients with $\mathrm{PBC}$ had not taken antibiotics for at least 2 months before entry. The patients were all UDCA-naïve. After entry, patients were administered UDCA using a daily dose of $13-15 \mathrm{mg} / \mathrm{kg}$. Faecal samples were collected again from 37 followed-up patients after 6 months of UDCA treatment. The biochemical response to UDCA was evaluated according to the PARIS II definition: serum AKP and aspartate aminotransferase $\leq 1.5$ upper limit of normal (ULN), with a normal bilirubin level. ${ }^{24}$ After UDCA treatment, 29/37 patients with PBC had an adequate response; $8 / 37$ patients had an inadequate clinical response. Age, gender and body mass index (BMI)-matched healthy controls were collected from volunteers as part of routine annual physical exams. The control group had to fulfil the following inclusion criteria: normal ranges of (a) liver and kidney function tests, (b) fasting blood glucose, blood lipids, urine and stools, BMI, and (c) an absence of hepatitis $\mathrm{B} / \mathrm{C}$ virus antigen. Subjects who received antibiotics within 2 months before sample collection were excluded. Written informed consent was obtained from each participant following protocols approved by the institutional review boards of Shanghai Jiao Tong University.

\section{Sample collection and DNA extraction}

Faecal samples were collected at the hospital and frozen at $-80^{\circ}$ C within 3 hours after sampling. DNA extraction was performed using a QIAamp Fast DNA Stool Mini Kit (Qiagen, California, USA). The concentration of bacterial DNA was measured using Nanodrop 2000 (Thermo Scientific, USA). Demographics and clinical variables were collected during the clinic visits.

\section{$16 \mathrm{~S}$ ribosomal RNA gene sequencing}

The V3-V4 region of the bacteria's 16S ribosomal RNA (rRNA) gene was amplified by PCR with barcode-indexed primers (338F and 806R), using FastPfu Polymerase. Amplicons were then purified by gel extraction (AxyPrep DNA GelExtraction Kit, Axygen Biosciences, Union City, California, USA) and were quantified using QuantiFluor-ST (Promega, USA). The purified amplicons were pooled in equimolar concentrations, and paired-end sequencing was performed using an Illumina MiSeq instrument (Illumina, San Diego, California, USA).

\section{Microbial analysis}

The 16S rRNA sequencing data were processed using the Quantitative Insights Into Microbial Ecology platform (V.1.9.1). ${ }^{25}$ Sequencing reads were demultiplexed and filtered. Operational taxonomic units (OTUs) were picked at 97\% similarity cut-off, and the identified taxonomy was then aligned using the Greengenes database (V.13.8). Chimeric sequences were identified and deleted. OTUs with a number of sequences $<0.005 \%$ of the total number of sequences were removed from the OTU table. ${ }^{26}$ After filtering, an average of 34661 reads per sample was obtained (min: 23 994; max: 42 940). In addition, rarefaction was performed on the OTU table to prevent methodological artefacts arising from varying sequencing depth. $\alpha$-Diversity was measured by species richness from the rarefied OTU table. $\beta$-Diversity was estimated by computing unweighted UniFrac and was visualised with principal coordinate analysis. In efforts to dissect possible species for OTUs, we performed MegaBLAST search to align the reads of OTUs against reference sequences in the National Center for Biotechnology Information (NCBI) 16S rRNA database.

\section{Imputed metagenomic analysis}

The metagenomes of gut microbiome were imputed from $16 \mathrm{~S}$ rRNA sequences with PICRUSt (Phylogenetic Investigation of Communities by Reconstruction of Unobserved States). ${ }^{27}$ This method predicts the gene family abundance from the phylogenetic information with an estimated accuracy of 0.8 . The closed OTU table was used as the input for metagenome imputation and was first rarefied to an even sequencing depth prior to the PICRUSt analysis. Next, the resulting OTU table was normalised by $16 \mathrm{~S}$ rRNA gene copy number. The gene content was predicted for each individual. Then the predicted functional composition profiles were collapsed into level 3 of KEGG (Kyoto Encyclopedia of Genes and Genomes) database pathways. Pathways present in $<10 \%$ of the samples were not included in the comparison analysis.

\section{Statistical analysis}

All statistical analyses were performed using $\mathrm{R}$ packages (V.2.15.3). For the comparison of continuous variables, Mann-Whitney U test (Kruskal-Wallis test for more than two 
groups) was used. For correlation analysis, Spearman's rank test was performed. Multiple hypothesis tests were adjusted using Benjamini and Hochberg false discovery rate (FDR), and significant association was considered below a FDR threshold of 0.05 . Logistic regression tests were used to regress the relative abundance of taxon against age, gender and BMI. The relative abundance was arcsine square root transformed before logistic regression test. A random forest model (randomForest 4.6-7 package) using the 12 genera signature was applied on the data from the PBC and control samples. To evaluate the discriminatory ability of the random forest model, operating characteristic curves (receiving operational curve, ROC) were constructed and area under curve (AUC) was calculated.

\section{RESULTS}

\section{Analysis of treatment-naive PBC}

We recruited a total of 79 subjects with PBC and 114 unrelated control subjects. All the patients were newly diagnosed with $\mathrm{PBC}$, and faecal samples in all cases were collected prior to initiation of UDCA treatment. After administration of a dose of 13$15 \mathrm{mg} / \mathrm{kg}$ daily for 6 months, 37 of the 79 patients provided additional stool samples. The demographic and clinical characteristics of PBC and controls are shown in table 1. Age, gender and BMI were comparable between $\mathrm{PBC}$ and healthy controls. As expected, there were considerable reductions of liver biochemistries and levels of IgM in the group after UDCA treatment compared with baseline (table 1). According to the time of enrolment, the first $60 \mathrm{PBC}$ and 80 controls were included in the exploration cohort, while the subsequent 19 $\mathrm{PBC}$ and 34 controls were included in the validation cohort (see online supplementary table S1).

\section{Gut microbial dysbiosis in treatment-naïve PBC}

The gut microbiota richness, measured by numbers of observed OTUs, was reduced in the 60 treatment-naïve individuals with $\mathrm{PBC}$ as compared with the data on the 80 healthy controls $(p=0.033$, Wilcoxon rank-sum test; figure $1 \mathrm{~A})$. We confirmed the decreased species richness after adjusting for age, gender and BMI $(p=0.038)$. However, the Shannon index which measures both richness and evenness was not significantly different between PBC and controls $(\mathrm{p}>0.05)$. An unweighted UniFrac-based principal coordinated analysis revealed that the overall microbial composition of PBC deviated from the healthy controls (PERMANOVAR, pseudo-F: 3.88, $\mathrm{p}=0.001$, figure $1 \mathrm{~B}$ ). Notably, we found that species richness can explain the differences along the first principal coordinate (figure 1C).

To investigate the specific changes of microbiota in samples from $\mathrm{PBC}$, we assessed the relative abundance of taxa in UDCA-naïve PBC and healthy controls. At the phylum level, Bacteroidetes spp were significantly decreased in PBC, whereas Fusobacteria and Proteobacteria spp were over-represented in $\mathrm{PBC}$ relative to controls (see online supplementary figure S1).

At the genus level, we observed 17 bacterial taxa that displayed different abundance between PBC and healthy individuals $\left(\mathrm{p}_{\mathrm{fdr}}<0.05\right.$, Wilcoxon rank-sum test. Twelve genera remained significant after correction for confounding variables including age, gender and BMI (see online supplementary table S2). Bacteroidetes spp, the most abundant genus in both groups, was decreased in PBC, compared with control group. Of the remaining taxa, three genera including Sutterella, Oscillospira and Faecalibacterium were enriched in healthy controls. Conversely, eight genera (Haemophilus, Veillonella, Clostridium, Lactobacillus, Streptococcus, Pseudomonas, Klebsiella and an unknown genus in the family of Enterobacteriaceae (Enterobacteriaceae,g)) were significantly increased in PBC (figure 2A). Notably, the unknown genus in the family of Enterobacteriaceae showed the strongest association with PBC $\left(p_{\mathrm{fdr}}=0.000046\right)$ and demonstrated a 6.4-fold increase in patients, compared with controls.

Spearman correlation test was performed to evaluate the relationships among the PBC-associated genera. Significant positive correlations were found in the following PBC-enriched genera: Enterobacteriaceae,g and Klebsiella $(\rho=0.64)$; Veillonella and Streptococcus $(\rho=0.55)$; Veillonella and Haemophilus $(\rho=0.65)$. In general, the genera enriched in PBC negatively correlated with genera enriched in the control subjects (figure 3), suggesting an antagonistic relationship between harmful and beneficial bacteria.

Table 1 Demographic characteristics of study subjects

\begin{tabular}{|c|c|c|c|c|c|}
\hline & \multicolumn{3}{|l|}{ PBC } & \multicolumn{2}{|l|}{ Healthy controls } \\
\hline & $\begin{array}{l}\text { Before UDCA } \\
(n=79)\end{array}$ & $\begin{array}{l}\text { After UDCA } \\
(n=37)\end{array}$ & p Valuet & $(n=114)$ & p Value* \\
\hline Age, median years (min-max) & $52(22-78)$ & $53(22-78)$ & & $47.5(25-65)$ & 0.12 \\
\hline Gender, female (\%) & $67(84.8 \%)$ & $36(97.3 \%)$ & & $91(79.8 \%)$ & 0.45 \\
\hline BMI, median $\mathrm{kg} / \mathrm{m}^{2}$ (min-max) & $22.6(17.6-29.0)$ & $22.5(17.6-29.0)$ & & $22.4(16.2-29.4)$ & 0.45 \\
\hline ALT, U/L, median (min-max) & $66(8-760.8)$ & $24(6.1-93)$ & $<0.0001$ & & \\
\hline AST, U/L, median (min-max) & $58(9.1-510)$ & $28(15-86)$ & $<0.0001$ & & \\
\hline AKP, U/L, median (min-max) & $197(46.9-1416)$ & $133(49-300)$ & $<0.0001$ & & \\
\hline GGT, U/L, median (min-max) & $188.5(13-1560)$ & $44(10-338)$ & $<0.0001$ & & \\
\hline $\mathrm{TB}, \mu \mathrm{mol} / \mathrm{L}$, median (min-max) & $14.3(5.1-250.3)$ & $12.3(4.1-48.8)$ & 0.33 & & \\
\hline $\operatorname{lgM}, g / L$, medain (min-max) & $2.965(0.79-9.6)$ & $2.32(0.36-6.16)$ & $<0.0001$ & & \\
\hline $\operatorname{lgG}, g / L$, medain (min-max) & $15.5(10.3-30.1)$ & $15.2(10.3-23.2)$ & 0.04 & & \\
\hline $\mathrm{AMA}, \pm, \mathrm{n}(+\%)$ & $77 / 2,97.5 \%$ & & & & \\
\hline gp210,, $\pm n(+\%)$ & $34 / 45,43.0 \%$ & & & & \\
\hline $\operatorname{sp} 100, \pm, n(+\%)$ & $8 / 71,10.1 \%$ & & & & \\
\hline
\end{tabular}



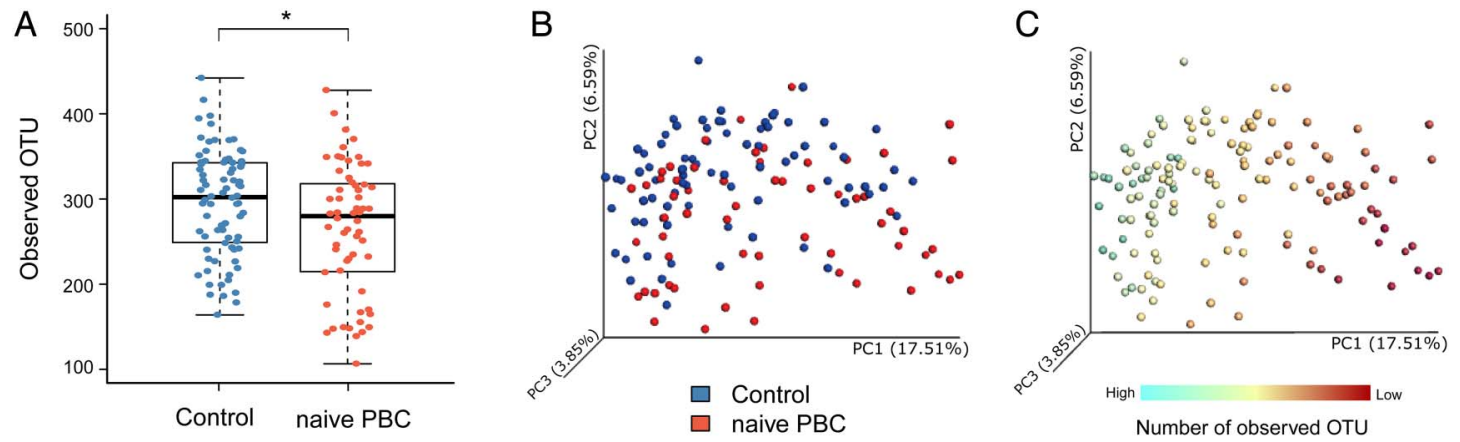

Figure 1 Changes of faecal microbial diversities in ursodeoxycholic acid treatment-naïve primary biliary cholangitis $(P B C)(n=60)$ compared with healthy controls $(n=80)$. (A) $\alpha$-Diversity, illustrated by microbiota richness (number of observed operational taxonomic unit (OTU)), was reduced in PBC $(p=0.033$, Wilcoxon rank-sum test). (B) Principal coordinate analysis (PCoA) of unweighted UniFrac analysis demonstrated that individuals with PBC were significantly different from healthy controls (pseudo-F: 3.88, $\mathrm{p}=0.001$, PERMANOVAR). (C) The same PCoA plot as (B), coloured by $\alpha$-diversity measured by number of observed OTU. Boxes represent the 25th-75th percentile of the distribution; the median is shown as a thick line in the middle of the box; whiskers extend to values with 1.5 times the difference between the 25th and 75 th percentiles. ${ }^{*} p<0.05$.
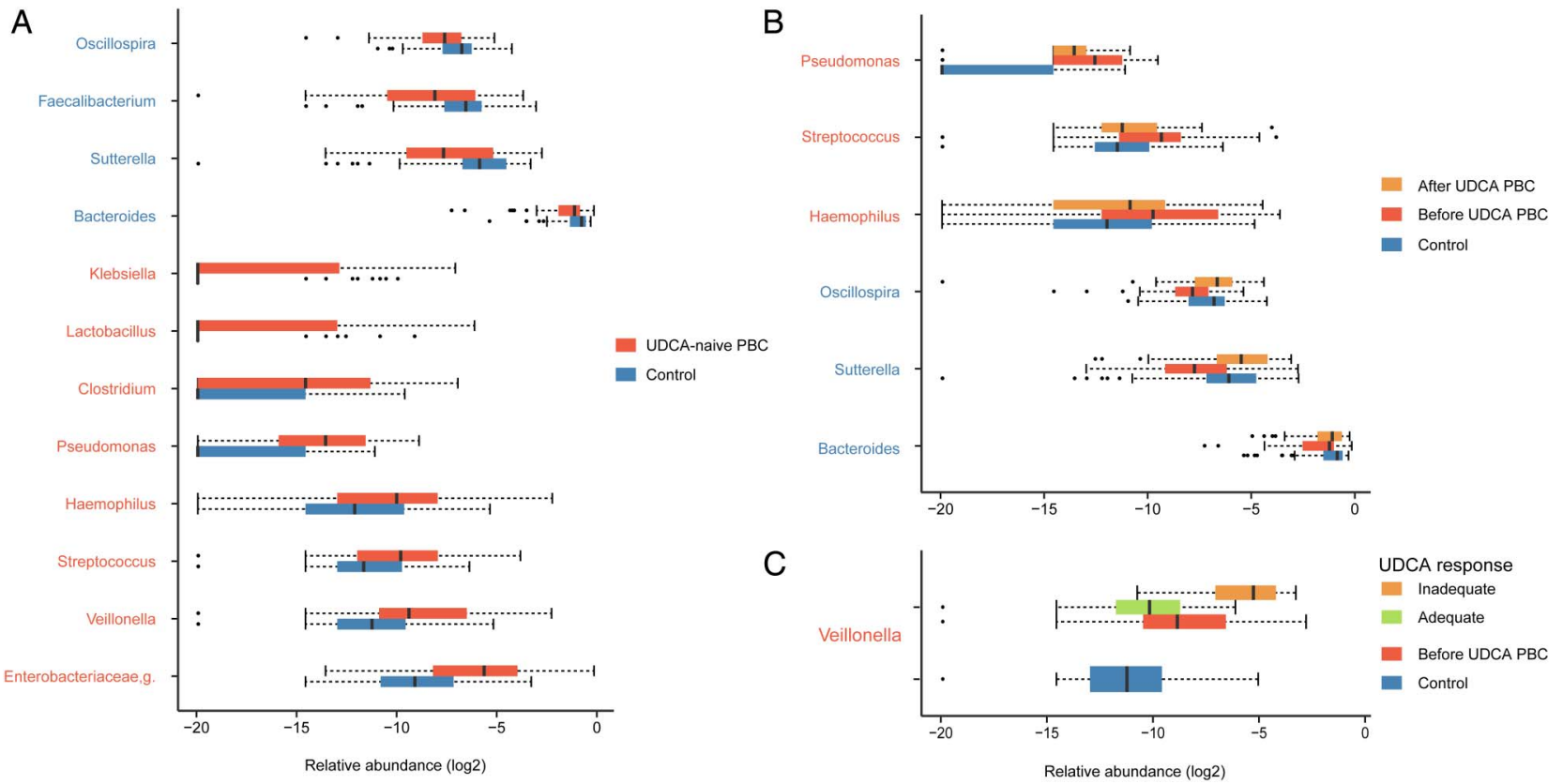

Figure 2 Microbiome alterations at the genus level in primary biliary cholangitis (PBC). (A) The relative abundance of 12 genera were significantly different between ursodeoxycholic acid (UDCA)-naïve PBC $(n=60)$ and healthy controls $(n=80)$ after correcting for confounding variables. (B) The microbiome composition was partially mitigated in UDCA-treated patients. Six of the PBC-associated genera were reversed in PBC after use of UDCA, compared with baseline ( $N=37, p<0.05$, paired Wilcoxon rank-sum test). (C) After UDCA treatment, the PBC-enriched genera Veillonella was more abundant in patients who demonstrated inadequate response than in those with adequate response $(n=8,29$, respectively; $p=0.0012$, Wilcoxon rank-sum test). The relative abundance of the genera is plotted on a logarithmic scale, and values of zero are assigned $1 \mathrm{e}-06$. Genera enriched in controls are in blue text, and genera over-represented in patients are in red text. Box plot illustration is provided in figure 1.

\section{Disease status discrimination with the 12-genera microbiome signature}

To explore the potential ability of the gut microbiome to discriminate PBC status, we constructed a random forest model based on the gut microbiome signature composed of 12 PBC-associated genera. The performance of the model was assessed using ROC analysis, achieving an AUC value of 0.86 (95\% CI 0.79 to 0.91 , figure 4 A). Subsequently, we validated the discriminatory power of the model using an independent group comprising $19 \mathrm{PBC}$ and 34 controls. The AUC was 0.84 in the validation cohort ( $95 \%$ CI 0.77 to 0.90 , figure $4 \mathrm{~B}$ ), confirming that the gut microbiome-based classifier is able to accurately distinguish $\mathrm{PBC}$ from controls.
UDCA treatment partially ameliorates gut dysbiosis of PBC

To determine the effect of UDCA therapy on microbial composition, we collected faecal samples from a subgroup of PBC before and after treatment $(\mathrm{n}=37)$. There was no significant alteration in the within-individual diversity in the samples from the UDCA-treated PBC relative to baseline samples (see online supplementary figure S2). While $\beta$-diversity using unweighted UniFrac did not show significant differences in the overall microbial composition between UDCA-naïve PBC and treated PBC, weighted UniFrac metric revealed a modest global difference between the two groups (weighted UniFrac, PERMANOVAR, pseudo-F: 2.87, $\mathrm{p}=0.026$ ). We then compared the relative abundance of PBC-associated taxa before and after treatment. 
Figure 3 Spearman correlations among the 12 primary biliary cholangitis (PBC)-associated genera in treatment-naïve PBC $(n=60)$ and healthy controls $(n=80)$. Overall microbial genera enriched in PBC (in red text) were negatively correlated with control-enriched genera (in blue text). Strong correlations were observed between

Enterobacteriaceae, $g$ and Klebsiella $(\rho=0.64)$; Veillonella and Streptococcus $(\rho=0.55)$; Veillonella and Haemophilus $(\rho=0.65)$.
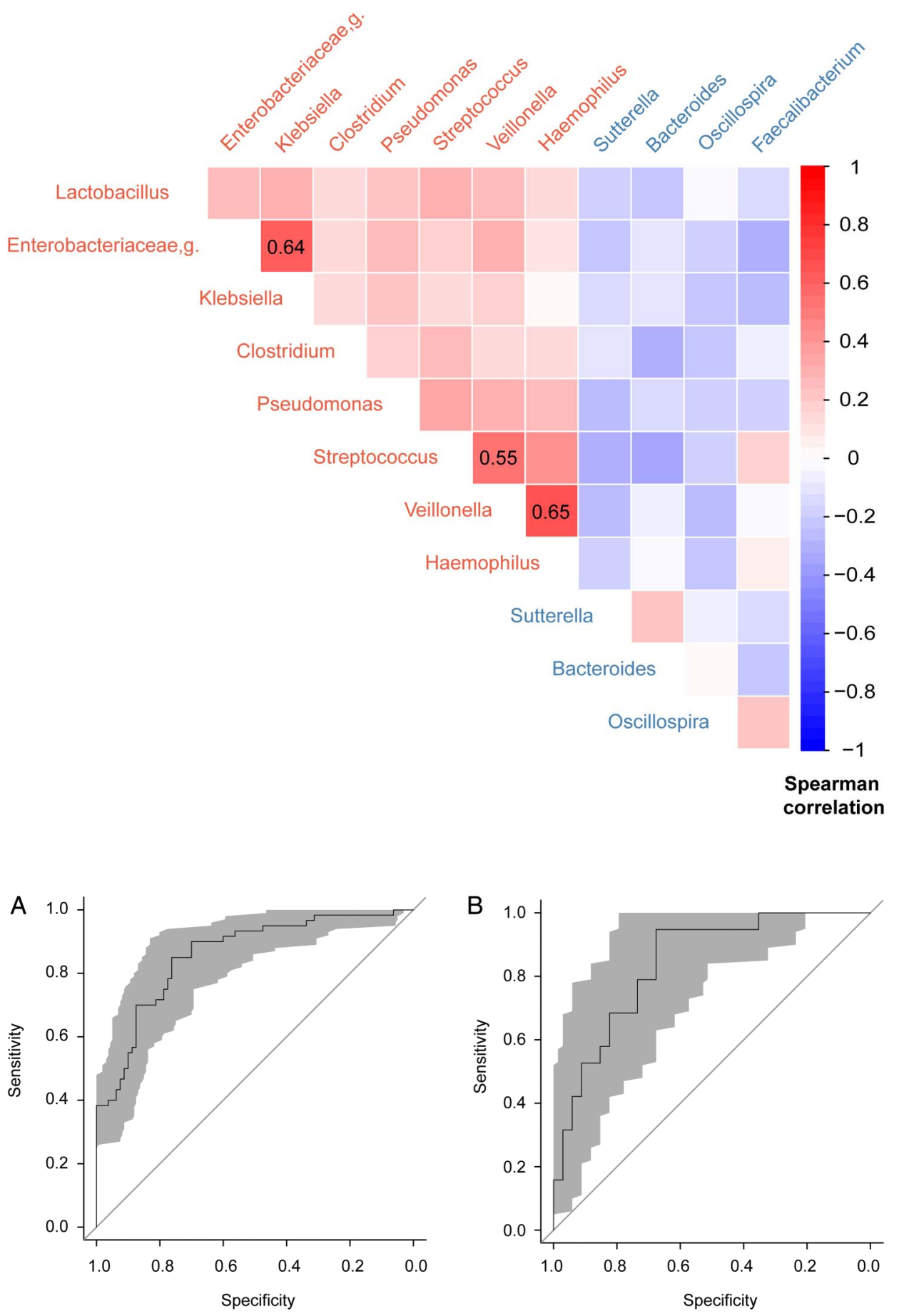

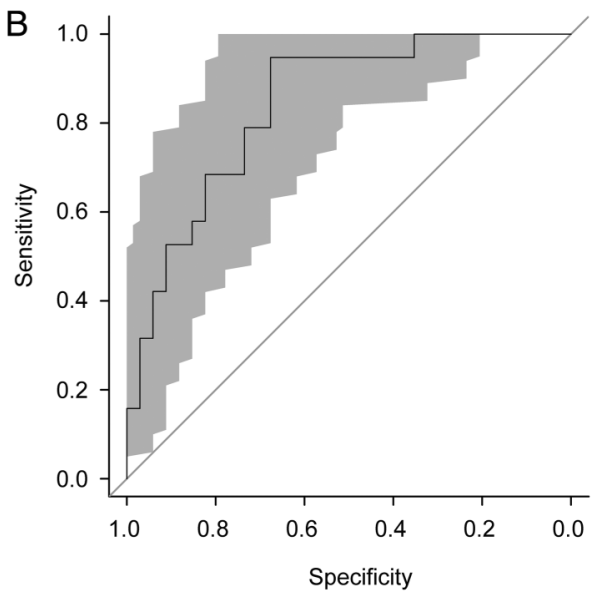

Figure 4 Gut microbiota signature can be used to discriminate between treatment-naïve patients from healthy controls. Receiving operational curve analysis was performed in (A) exploration data (primary biliary cholangitis (PBC): 60; control: 80, area under curve $(A \cup C)=0.86)$ and in $(B)$ validation data (PBC: 19; control: 34, $A \cup C=0.84)$, respectively. Diagonal lines represent random classification $(\mathrm{AUC}=0.5)$.
Remarkably, the differences in the genera identified between the UDCA-untreated patients and healthy controls were partially reversed in the PBC subjects after 6 months of UDCA therapy. Among the PBC-enriched genera, Haemophilus spp, Streptococcus spp and Pseudomonas spp decreased after UDCA treatment $(\mathrm{p}<0.05$, paired Wilcoxon rank-sum test). Of the four genera enriched in healthy controls, three (Bacteroidetes spp, Sutterella spp and Oscillospira spp) expanded in the posttreatment group (figure $2 \mathrm{~B}$ and see online supplementary table S3). Although the genus Veillonella spp that was enriched in samples from the PBC did not decrease in patients after treatment, it was significantly more prevalent in treated patients with an inadequate response to UDCA than those who demonstrated good improvement $(p=0.0012$, Wilcoxon rank-sum test, figure
2C). Thus, the microbial dysbiosis in PBC could be partially alleviated by UDCA treatment, and the microbiome had the potential to evaluate the treatment outcome.

Associations between clinical variables and gut microbiota We investigated the effects of subphenotypes on the gut microbiota in treatment-naïve PBC. The patients were stratified into early, moderate and advanced stages based on serum levels of total bilirubin (TB) and albumin. ${ }^{28}$ There was no significant difference in patients at different stages with respect to $\alpha$-diversity, $\beta$-diversity or relative abundance of associated PBC taxa (see online supplementary figures S3A and S4A). We then classified patients according to the status of anti-gp210 antibody, which is indicative of disease progression in PBC. ${ }^{29}$ 
gp210-positive PBC had reduced microbiome richness, when compared with gp210-negative patients, although the analysis did not reach statistical significance (see online supplementary figure S3B). The overall microbial composition did not differ between gp210-positive and gp210-negative patients (see online supplementary figure S4B). Importantly, Faecalibacterium spp, the control-enriched genus, was significantly more abundant in gp210-negative patients than gp210-positive patients $(\mathrm{p}=0.002$, see online supplementary figure S5). The finding withstood logistic regression analysis adjusted for age, gender and BMI (adjusted $\mathrm{p}=0.008$ ). In fact, $F$. prausnitzii is the only known species in the Faecalibacterium genus. We next examined whether gender bias in $\mathrm{PBC}$ was associated with gut microbiome. Within-individual or between-individual diversity was not significantly related to gender status in our cohort (see online supplementary figures S3C and S4C). Of note, Pseudomonas, Streptococcus and Haemophilus were more abundant in female patients with $\mathrm{PBC}$ than male patients with $\mathrm{PBC}$ (uncorrected $\mathrm{p}=0.019,0.031$ and 0.040 , respectively, Wilcoxon rank-sum test, see online supplementary figure $\mathrm{S6}$ ). In addition, relative to control males, control females demonstrated a significant enrichment in an abundance of Haemophilus spp $(p=0.0056)$. Remarkably, these three gender-associated genera were the same PBC-enriched genera that decreased in PBC after UDCA treatment.

The Spearman correlation test was used to investigate the correlation between the relative abundance of the signature PBC genera and clinical indices (see online supplementary figure S7). We observed a positive correlation between Lactobacillus and conjugated bilirubin (CB) $(\rho=0.32, p=0.005)$. There was also a trend towards a positive correlation between the Klebsiella genus and levels of TB, $\gamma$-glutaryltransferase and $\mathrm{CB}(\rho=0.31$, $\mathrm{p}=0.006 ; \rho=0.29, \mathrm{p}=0.011 ; \rho=0.26, \mathrm{p}=0.026$; respectively). Only the correlation between Klebsiella and TB remained significant after correcting for the confounding variables $(p=0.013)$, but did not withstand multiple testing correction.

\section{Microbial functions altered in PBC}

To characterise the functional alterations of the gut microbiome in $\mathrm{PBC}$, we predicted the functional composition profiles from 16S rRNA sequencing data with PICRUSt in all UDCA-naïve
PBC and controls (PBC: 79; control: 114). After correcting for the effects of age, gender and BMI, we found that multiple KEGG (level 3) categories were disturbed in PBC. The pathways enriched in $\mathrm{PBC}$ highlighted bacterial invasion of epithelial cells, peroxisome proliferator-activated receptors (PPAR) signalling pathway and caprolactam degradation (see online supplementary figure S8A). In contrast, the gut microbiome of controls was characterised by over-representation of proteins involved in metabolism and the biosynthesis of amino acids (eg, alanine, aspartate and glutamate) as well as $\alpha$-linoleic acid metabolism (see online supplementary figure S8B). Strikingly, bacterial invasion of epithelial cells was the most significantly altered pathway in the predicted $\mathrm{PBC}$ metagenomes after adjusting for confounding variables (figure $5 \mathrm{~A}$ ).

\section{Bacteria in the family Enterobacteriaceae correlate with the functional module}

Intriguingly, the abundance of bacterial invasion of epithelial cells was positively correlated with two genera in the Enterobacteriaceae family (Enterobacteriaceae,g and Klebsiella; $\rho=0.95$ and 0.57 , respectively; figure $5 \mathrm{~B}, \mathrm{C})$. We further examined the two genera at the OTU level. Three OTUs (OTU1, OTU2, OTU3) accounted for more than $90 \%$ of the sequences in the Enterobacteriaceae,g. All three OTUs were differentially abundant between treatment-naïve patients and healthy controls $(\mathrm{p}=1.51 \mathrm{E}-5,1.57 \mathrm{E}-7$ and $5.65 \mathrm{E}-6$, respectively; Wilcoxon rank-sum test). Moreover, the three OTUs demonstrated a positive correlation with bacterial invasion of epithelial cells $(\rho=0.58,0.78$ and 0.62 , respectively; see online supplementary figure $\mathrm{S} 9 \mathrm{~A}-\mathrm{C}$ ). The results remained significant when confounders for age, gender and BMI were taken into account. The sequences of OTU1, OTU2 and OTU3 matched with several highly similar species such as Klebsiella pneumonia, Escherichia coli and Enterobacter cloacae ( $\geq 99 \%$ of sequence identity, see online supplementary table S4). Nevertheless, only one OTU (OTU4) was responsible for the association of the genus Klebsiella with PBC status and its correlation with the pathway $(p=1.43 \mathrm{E}-4$, Wilcoxon rank-sum test; $\rho=0.57$; see online supplementary figure S9D). K. pneumonia and $K$. variicola had a nearly perfect match with the read of OTU4 (see online supplementary table S4).
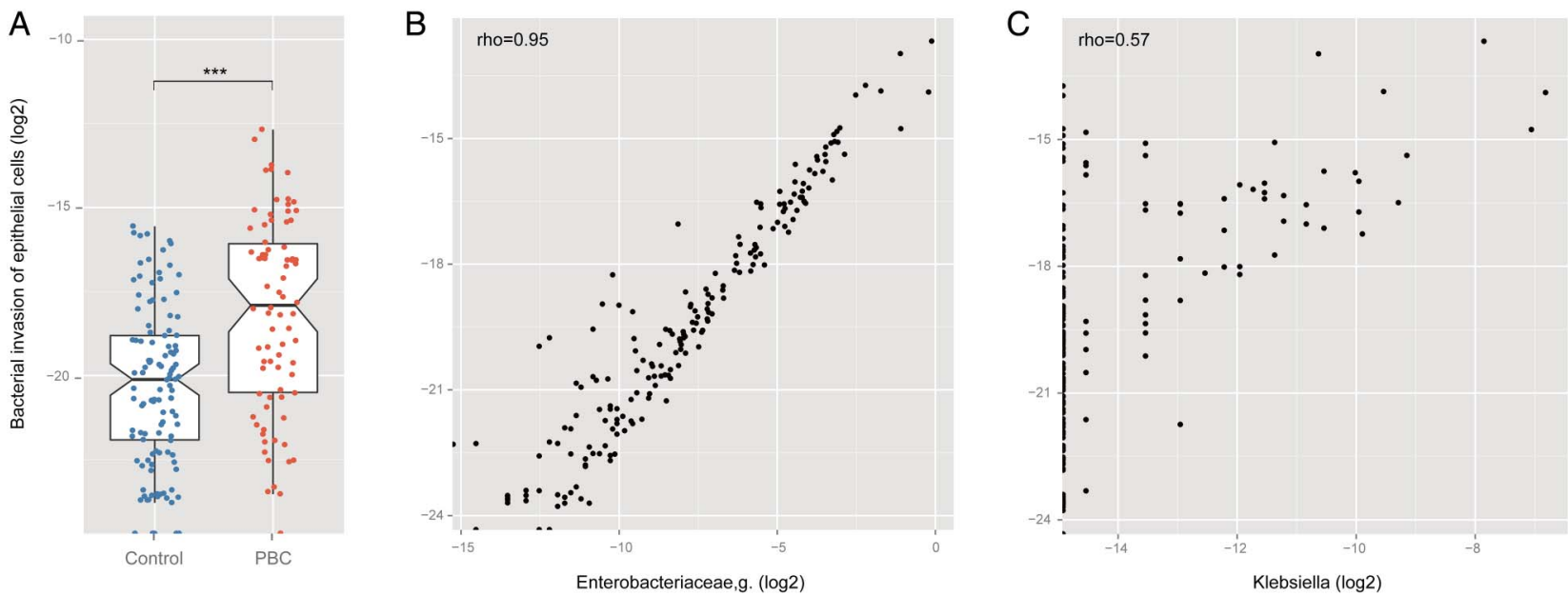

Figure 5 The predicted functional module, bacterial invasion of epithelial cells, altered in ursodeoxycholic acid (UDCA)-naïve primary biliary cholangitis (PBC). (A) A significant occurrence of differentially abundant bacterial invasion of epithelial cells pathway between UDCA-naïve PBC $(n=79)$ and healthy controls $(n=114),\left(p_{f d r}=0.0003\right)$. False discovery rate (FDR)-corrected $p$ values were calculated after adjusting for age, gender and body mass index. (B) and (C) The two PBC-enriched genera in the family of Enterobacteriaceae were highly correlated with bacterial invasion of epithelial cells $\left(\rho=0.95,0.57\right.$, respectively, Spearman's rank test). ${ }^{* * *} p<0.001$. 


\section{DISCUSSION}

In the current study, we applied a $16 \mathrm{~S}$ rRNA sequencing approach to a large, unique UDCA-naïve PBC cohort in addition to a longitudinal study of a subset of patients analysed before and after UDCA therapy. In the cross-sectional study, we demonstrated that PBC was associated with altered composition and function of gut microbiota, as well as a moderately lower level of diversity. In the prospective study, 6 months of UDCA use was found to partially mitigate the microbial dysbiosis. UDCA modified the composition of the gut microbiome, while not significantly affecting the microbial diversity.

In our study, the gut microbiome of PBC reflected reduced species richness as well as a significant shift in the overall microbial diversity. No obvious differences were found in within-individual or between-individual diversity in a recent study of microbiomes conducted on a small cohort of PBC. ${ }^{21}$ In our study, we included only treatment-naive patients, whereas the earlier study did not. Bile acids have a strong effect on microbial composition. The use of UDCA could modify the composition of the microbiome which is consistent with the results of the present study, and thus affect the data. Therefore, our findings underscore the requirement for a large cohort and an optimised study design to ensure adequate statistical power for capturing microbial diversities.

We should also note that the difference of within-sample diversity between PBC and controls was modest in our data. Of interest, gp210-positive patients showed relatively lower species richness than gp210-negative patients, which suggested that the reduced microbiome richness in $\mathrm{PBC}$ could be attributed to gp210-positive patients. It has been suggested that anti-gp210 antibody reflects disease severity in PBC, associated with more severe interface hepatitis and hepatic failure progression. ${ }^{29}$

PBC microbial dysbiosis was characterised by altered abundances of 12 genera. The combination of the 12 associated taxa discriminated patients from healthy controls with high accuracy and was successfully verified in a small independent cohort. We noted that gut microbiome-based analysis displayed similar predictive ability for the disease as the classifier based on PBC-associated genetic variants which had an AUC of $0.82,{ }^{30}$ implying that the microbial signature identified may be a potentially powerful tool for disease prediction and that integrating genetic markers and microbial profiles may further improve the discriminative ability.

Interestingly, the eight PBC-enriched genera were relatively rare in the normal human gut. It is recognised that potentially pathogenic bacteria that are normally present in low abundance can thrive and contribute to inflammation or autoimmunity under inflammatory conditions. An unknown genus in the family of Enterobacteriaceae revealed the most significant association with PBC. Indeed, the major autoantigens of human PBC are readily found in all bacterial species that belong to the Enterobacteriaceae family. We posit that AMA may be induced by rough mutants of the members of the Enterobacteriaceae. ${ }^{31}$ Klebsiella, also a genus in the family of Enterobacteriaceae, displayed a positive correlation with serum levels of bilirubin, which is an important prognostic factor of PBC. Klebsiella microbes have been implicated in the pathogenesis of autoimmune disorders including ankylosing spondylitis and Crohn's disease (CD), likely due to cross-reactivity and molecular mimicry. ${ }^{32}$ The fact that Klebsiella organisms are associated with urinary tract infection supports this view. ${ }^{33}$ Recurrent urinary tract infections are a risk factor for the development of PBC. ${ }^{34}$ Veillonella genus has recently been associated with PSC and recurrent $\mathrm{CD} .^{13} 35$ Veillonella was also found in severe inflammatory conditions including endocarditis and cystic fibrosis. ${ }^{3637}$ Interestingly, the presence of Veillonella positively correlated with the presence of Streptococcus spp and Haemophilus spp, both of which were increased in patients with CD and liver cirrhosis. In the study herein, decreases in the abundance of Faecalibacterium, Bacteroides, Sutterella and Oscillospira spp were found in PBC. Remarkably, gp210-positive PBC had even lower abundance of Faecalibacterium than gp210-negative PBC. F. prausnitzii, the major representative of the Faecalibacterium genus, is an important component of a healthy human gut microbiome. Moreover, F. prausnitzii is an important supplier of butyrate that has been suggested to have an anti-inflammatory function within the gut mucosa. Thus, F. prausnitzii may play an important role in maintaining gut health.

Microbial dysbiosis in PBC was partially relieved after UDCA treatment. Since bile acids have emerged as important regulators of intestinal microbiota, it will be interesting to investigate the influence of obeticholic acid on microbiome, which was recently approved by Food and Drug Administration (FDA) for the treatment of PBC. It is also worth mentioning that PBC-enriched genera that were reversed after UDCA treatment were significantly enriched in female patients with $\mathrm{PBC}$ compared with male patients with PBC. Murine model studies of type I diabetes indicate that gender bias may be influenced by the gut microbial community through its interaction with sex hormones, although the underlying mechanisms are poorly characterised. ${ }^{38} 39$ In contrary to $\mathrm{PBC}$, no significant difference in gut microbiome has been observed between patients with PSC taking UDCA and those not. ${ }^{14}$ This coincides with the distinct therapeutic efficacy of UDCA in PBC and PSC. Of note, the comparison was conducted between patients with and without UDCA treatment in the PSC study, which is different from our study design.

Analysis of inferred PBC metagenome identified the strongest association with the bacterial invasion of epithelial cells pathway in which pathogenic bacteria may cross epithelial barriers, colonise cells and invade internal tissues. ${ }^{40}$ Intriguingly, the enrichment of bacterial invasion of epithelial cells could be attributed to the significantly increased abundances of two genera in the family of Enterobacteriaceae, the sequences of which had high similarity with the bacteria such as E. coli, K. pneumonia and E. cloacae. In fact, several bacteria from Enterobacteriaceae family, especially E. coli and K. pneumonia, have been found to be the most common bacteria that translocate into the mesenteric lymph nodes and liver, and cause bacterial infection in patients with liver cirrhosis. ${ }^{41}{ }^{42}$ Indeed, many of the PBC-associated microbes in our study have been identified in the previous study of the microbiomes of liver cirrhosis. ${ }^{16}$

Although our investigations attempt to provide a comprehensive insight into the potential contribution of the gut microbiome in PBC, there are several limitations to be addressed in future studies. First, we were not able to observe variations among patients at different disease stages, probably due to a relatively small number of patients at the moderate or advanced stage, as most patients in our cohort were new-onset PBC. Second, our pathway analysis was based on the inferred metagenome from 16S rRNA sequence. Although inference of a metagenome approach (PICRUSt) has been commonly used in 16S rRNA studies, short-gun sequencing for metagenomics and metatranscriptomics may reveal more accurate microbial community composition and function. Third, the mucosal microbiota is beyond the scope of the current study; stool samples are more readily obtained, but faecal microbiota cannot fully reflect the profiles of mucosal microbiota. ${ }^{43}$ Fourth, homogenisation of samples was not performed before DNA extraction in our study. 
A mechanical procedure (eg, bead beating) of faecal samples can help efficient lysis and homogenising, thus improving the yield and quality of bacterial DNA. ${ }^{44}$ Fifth, although age, gender and BMI were adjusted in our analysis, our results could be influenced by confounding effects such as environmental and dietary factors. Finally, it is important to note that microbiome analysis must include studies of the 'virome' and 'fungiome' in addition to considerations of vaginal flora, particularly due to the gender bias of PBC. Nonetheless, our comprehensive investigation of the gut microbiome in PBC reveals compositional and functional dysbiosis in patients that are partially alleviated by UDCA. The identified PBC microbial signature needs further validation in larger and independent cohorts.

\section{Author affiliations}

'Division of Gastroenterology and Hepatology, Key Laboratory of Gastroenterology and Hepatology, Ministry of Health, State Key Laboratory for Oncogenes and Related Genes, Renii Hospital, School of Medicine, Shanghai Jiao Tong University; Shanghai Institute of Digestive Disease, Shanghai, China

${ }^{2}$ Department of Health Manage Center, School of Medicine, Renji Hospital, Shanghai Jiao Tong University, Shanghai, China

${ }^{3}$ School of Life Sciences and Biotechnology, Shanghai Jiao Tong University, Shanghai, China

${ }^{4}$ Department of Pathology, Emory University School of Medicine, Atlanta, Georgia, USA

${ }^{5}$ Division of Rheumatology, Department of Medicine, Allergy and Clinical Immunology, University of California at Davis, Davis, California, USA

Acknowledgements The authors are grateful for all the subjects who participated in the study.

Contributors XM, MEG and RT designed and supervised the project. XM, J-YF and RT obtained funding. QW, FY, QM and DQ performed clinical diagnosis and treatment. YW, YL, WC, QC and ZF collected samples. YW, YL, ML, XJ and JZ contributed to data collection. RT, YW, YL and MW performed bioinformatics and statistical analysis, and interpreted data. RT, YW and YL drafted the manuscript. XM, MEG and AA revised the manuscript for important content.

Funding This work was supported by the National Natural Science Foundation of China grants (\# 81325002 and 81620108002 to XM; \#81421001 to J-YF; \# 81400608 and 81570469 to RT); the Innovation Program of Shanghai Jiao Tong University grant (\# YG2014MS43 to RT) and Shanghai Municipal Education Commission-Gaofeng Clinical Medicine Grant Support (\#20161311 to RT).

Competing interests None declared.

Ethics approval Renji Hospital, School of Medicine, Shanghai Jiao Tong University.

Provenance and peer review Not commissioned; externally peer reviewed.

\section{REFERENCES}

1 Griffiths L, Dyson JK, Jones DE. The new epidemiology of primary biliary cirrhosis. Semin Liver Dis 2014;34:318-28.

2 James OF, Bhopal R, Howel D, et al. Primary biliary cirrhosis once rare, now common in the United Kingdom? Hepatology 1999;30:390-4.

3 Kaplan MM, Gershwin ME. Primary biliary cirrhosis. N Engl J Med 2005;353:1261-73.

4 Carey EJ, Ali AH, Lindor KD. Primary biliary cirrhosis. Lancet 2015;386:1565-75.

5 Juran $\mathrm{BD}$, Lazaridis KN. Environmental factors in primary biliary cirrhosis. Semin Liver Dis 2014;34:265-72.

6 Seldin MF. The genetics of human autoimmune disease: a perspective on progress in the field and future directions. J Autoimmun 2015:64:1-12.

7 Wang $\mathrm{YH}$, Yang W, Yang JB, et al. Systems biologic analysis of $\mathrm{T}$ regulatory cells genetic pathways in murine primary biliary cirrhosis. J Autoimmun 2015;59:26-37.

8 Webb GJ, Hirschfield GM. Using GWAS to identify genetic predisposition in hepatic autoimmunity. I Autoimmun 2016;66:25-39.

9 Webb GJ, Siminovitch KA, Hirschfield GM. The immunogenetics of primary biliary cirrhosis: a comprehensive review. J Autoimmun 2015;64:42-52.

10 Rooks MG, Garrett WS. Gut microbiota, metabolites and host immunity. Nat Rev Immunol 2016;16:341-52.

11 Marchesi JR, Adams DH, Fava F, et al. The gut microbiota and host health: a new clinical frontier. Gut 2016:65:330-9.

$12 \mathrm{Li}$ J, Jia H, Cai X, et al. An integrated catalog of reference genes in the human gut microbiome. Nat Biotechnol 2014:32:834-41.

13 Kummen M, Holm K, Anmarkrud JA, et al. The gut microbial profile in patients with primary sclerosing cholangitis is distinct from patients with ulcerative colitis without biliary disease and healthy controls. Gut 2016. doi:10.1136/gutjnl-2015-310500. [Epub ahead of print 17 Feb 2016]

14 Sabino J, Vieira-Silva S, Machiels K, et al. Primary sclerosing cholangitis is characterised by intestinal dysbiosis independent from IBD. Gut 2016;65:1681-9.

15 Leung C, Rivera L, Furness JB, et al. The role of the gut microbiota in NAFLD. Nat Rev Gastroenterol Hepatol 2016;13:412-25.

16 Qin N, Yang F, Li A, et al. Alterations of the human gut microbiome in liver cirrhosis. Nature 2014:513:59-64.

$17 \mathrm{Ma} \mathrm{HD}$, Wang YH, Chang $\mathrm{C}$, et al. The intestinal microbiota and microenvironment in liver. Autoimmun Rev 2015;14:183-91.

18 Henao-Mejia J, Elinav $\mathrm{E}$, Thaiss $\mathrm{CA}$, et al. Role of the intestinal microbiome in liver disease. J Autoimmun 2013;46:66-73.

19 Wahlström A, Sayin SI, Marschall HU, et al. Intestinal crosstalk between bile acids and microbiota and its impact on host metabolism. Cell Metab 2016;24:41-50.

20 Ridlon JM, Kang DJ, Hylemon PB, et al. Bile acids and the gut microbiome. Curr Opin Gastroenterol 2014;30:332-8.

21 Lv LX, Fang DQ, Shi D, et al. Alterations and correlations of the gut microbiome, metabolism and immunity in patients with primary biliary cirrhosis. Environ Microbiol 2016;18:2272-86.

22 Lindor KD, Gershwin ME, Poupon R, et al. Primary biliary cirrhosis. Hepatology 2009:50:291-308.

23 Chazouillères 0 , Wendum $D$, Serfaty L, et al. Primary biliary cirrhosis-autoimmune hepatitis overlap syndrome: clinical features and response to therapy. Hepatology 1998:28:296-301.

24 Corpechot C, Chazouillères O, Poupon R. Early primary biliary cirrhosis: biochemical response to treatment and prediction of long-term outcome. J Hepatol 2011;55:1361-7

25 Kuczynski J, Stombaugh J, Walters WA, et al. Using QIIME to analyze 16S rRNA gene sequences from microbial communities. Curr Protoc Microbiol 2012; Chapter 1: Unit 1E.5.

26 Navas-Molina JA, Peralta-Sánchez JM, González A, et al. Advancing our understanding of the human microbiome using QIIME. Meth Enzymol 2013; 531:371-444.

27 Langille MG, Zaneveld J, Caporaso JG, et al. Predictive functional profiling of microbial communities using 16S rRNA marker gene sequences. Nat Biotechnol 2013;31:814-21.

28 ter Borg PC, Schalm SW, Hansen BE, et al. Prognosis of ursodeoxycholic Acid-treated patients with primary biliary cirrhosis. Results of a 10-yr cohort study involving 297 patients. Am J Gastroenterol 2006:101:2044-50.

29 Nakamura $\mathrm{M}$, Kondo $\mathrm{H}$, Mori T, et al. Anti-gp210 and anti-centromere antibodies are different risk factors for the progression of primary biliary cirrhosis. Hepatology 2007:45:118-27.

30 Tang $\mathrm{R}$, Chen $\mathrm{H}$, Miao $\mathrm{Q}$, et al. The cumulative effects of known susceptibility variants to predict primary biliary cirrhosis risk. Genes Immun 2015;16:193-8.

31 Stemerowicz R, Hopf U, Möller B, et al. Are antimitochondrial antibodies in primary biliary cirrhosis induced by R(rough)-mutants of enterobacteriaceae? Lancet 1988;2:1166-70.

32 Rashid T, Ebringer A. Autoimmunity in rheumatic diseases is induced by microbial infections via crossreactivity or molecular mimicry. Autoimmune Dis 2012;2012:539282.

33 Paczosa MK, Mecsas J. Klebsiella pneumoniae: going on the offense with a strong defense. Microbiol Mol Biol Rev 2016;80:629-61.

34 Smyk DS, Bogdanos DP, Kriese $S$, et al. Urinary tract infection as a risk factor for autoimmune liver disease: from bench to bedside. Clin Res Hepatol Gastroenterol 2012;36:110-21

35 De Cruz P, Kang S, Wagner J, et al. Association between specific mucosa-associated microbiota in Crohn's disease at the time of resection and subsequent disease recurrence: a pilot study. J Gastroenterol Hepatol 2015;30:268-78.

36 Rovery C, Etienne A, Foucault C, et al. Veillonella montpellierensis endocarditis. Emerg Infect Dis 2005:11:1112-14.

37 Coburn B, Wang PW, Diaz Caballero J, et al. Lung microbiota across age and disease stage in cystic fibrosis. Sci Rep 2015;5:10241.

38 Yurkovetskiy L, Burrows M, Khan AA, et al. Gender bias in autoimmunity is influenced by microbiota. Immunity 2013;39:400-12.

39 Markle JG, Frank DN, Adeli K, et al. Microbiome manipulation modifies sex-specific risk for autoimmunity. Gut Microbes 2014;5:485-93.

40 Ribet $\mathrm{D}$, Cossart P. How bacterial pathogens colonize their hosts and invade deeper tissues. Microbes Infect 2015;17:173-83.

41 Tuomisto S, Pessi T, Collin P, et al. Changes in gut bacterial populations and their translocation into liver and ascites in alcoholic liver cirrhotics. BMC Gastroenterol 2014;14:40.

42 Wiest R, Lawson M, Geuking M. Pathological bacterial translocation in liver cirrhosis. J Hepatol 2014:60:197-209.

43 Parthasarathy G, Chen J, Chen X, et al. Relationship Between microbiota of the colonic mucosa vs feces and symptoms, colonic transit, and methane production in female patients with chronic constipation. Gastroenterology 2016;150:367-79.e1.

44 Smith B, Li N, Andersen AS, et al. Optimising bacterial DNA extraction from faecal samples: comparison of three methods. Open Microbio/ J 2011;5:14-17. 